\title{
Condition-based Maintenance Optimization of Degradable Systems
}

\author{
Shuaichong Wei \\ Mechanical Engineering Department, \\ Laval University, Quebec, Canada. \\ E-mail: shuaichong.wei.1@ulaval.ca \\ Mustapha Nourelfath \\ Mechanical Engineering Department, \\ Laval University, Quebec, Canada. \\ Corresponding author: mustapha.nourelfath@gmc.ulaval.ca \\ Nabil Nahas \\ Département d'Administration, \\ Université de Moncton, Moncton (NB), Canada. \\ E-mail: nabil.nahas@umoncton.ca
}

(Received on September 26, 2021; Accepted on December 17, 2021)

\begin{abstract}
This paper develops a mathematical model for condition-based maintenance optimization of multi-state systems. The majority of the existing literature on maintenance optimization assume that there is no additional cost incurred because of side effects of equipment degradation. Nevertheless, as the operating cost increases with equipment age and degradation, it is important to consider the degradation side effects in the maintenance decision-making process. An important feature of the proposed model lies in the fact that it incorporates side effect of degradation process into condition-based preventive maintenance optimization. We develop a continuous-time discrete-state Markov chain model describing the deterioration stochastic process of a single component. The component is modeled as a multi-state system, where each discrete state is characterized by a degradation level. Numerical examples show the importance of considering such side effect costs when optimizing the choice of maintenance policy. The proposed model is extended to deal with multi-state series systems. Using an example of a series system with two components, it is shown that preventive maintenance and side effect costs should not be optimized for each component individually, but from the perspective of the series system as a whole.
\end{abstract}

Keywords- Maintenance, Degradation side effects, Multi-state systems, Markov chains, Optimization.

\section{Introduction}

As industrial devices are generally designed to operate for long periods, their performance is subject to progressive degradation until failure occurrence. During the last decades, degradation models have received a lot of attention for the analysis and design of highly reliable equipment (Eda et al., 1980; Pan and Chen, 2015). Several physical failures of engineering systems can be linked to a more or less complex degradation phenomenon (Liu et al., 2016). The majority of systems are subject to an observable degradation process. The attributes of a degraded state can be measured over time, via indicators that change with the age of the equipment. Actually, the degradation is manifested by many signs of oldness, such as noise, changes in temperature, vibration, pressure, product quality, energy consumption, and air pollution. The development of accurate real-time sensing techniques and monitoring software have facilitated the detection of failure precursors and the forecast of degradation trends. This technological advancement has also facilitated the use of continuous-time stochastic models to characterize the degradation process (Lam and Banjevic, 
2015). Such continuous-time modeling is suitable for the application of condition-based preventive maintenance as an efficient strategy to prevent random failures.

The objective of any maintenance strategy is to find the best compromise between corrective and preventive actions. Compared to classical age-based and block-based strategies (Barlow and Hunter, 1960), condition-based preventive maintenance is usually more effective in reducing the total cost and increasing the system reliability and availability (Wu and Ryan, 2010; Liu et al., 2017). This advantage is due to the fact that it relies on real-time information to determine the system health. There is a substantial literature dealing with the optimal replacement of continuously degrading systems (Flory et al., 2015), and the prognostic models to characterize the physical degradation phenomenon (Kang et al., 2020). It is possible to distinguish between two classes of the degradation states, namely discrete and continuous states (Liu et al., 2014; Kang et al., 2020). Considering continuous degradation states, a vast literature has considered stochastic models (Ye and Xie, 2015), based on Wiener process (Ye et al., 2013; Ye et al., 2015), Gamma process (AbdelHameed, 1975; Kallen and Noortwijk, 2005), and inverse Gaussian process (Wang and Xu, 2010; Peng, 2015). Using a (continuous stochastic) Wiener process, a condition-based maintenance policy has been developed in Liu et al. (2017) for a degrading system with age and state dependent operating cost. The literature using discrete degradation states has been based on Markov chain models (Yin et al., 2013; Fitouhi et al., 2017), with each state characterizing a level of degradation. The estimated performance measures are generally related to availability (Yin et al., 2013), throughput (Fitouhi et al., 2017), or long-run cost rate (Liao, 2013). Some studies have also considered the problem of value-based maintenance policy (Marais and Saleh, 2009; Rosqvist et al., 2009). In the context of production planning, other papers have considered degradations in quality (Tagaras, 1988; Yeung et al., 2007; Xiang, 2013; Nourelfath et al., 2016; Fakher et al., 2017; Kim and Sarkar, 2017; Gouiaa-Mtibaa et al., 2018), energy consumption (Jeon et al., 2015; Hoang et al., 2017; Zhou et al., 2020), and carbon dioxide $\left(\mathrm{CO}_{2}\right)$ emission (Ba et al., 2016; Hajej et al, 2017; Singh et al., 2019). There is a link between energy consumption, $\mathrm{CO}_{2}$ emission, and the production rate. The relationships between the processing time, power energy consumption and the carbon emissions have been illustrated in An et al. (2020) for dry milling processes.

It is important to consider the degradation side effects in the maintenance decision making process. In reality, degradation can be accompanied by side effects due to poor quality of a fraction of produced items, increased energy consumption and $\mathrm{CO}_{2}$ emission. However, the majority of the existing literature on maintenance optimization assume that there is no additional cost incurred because of side effects of equipment degradation. To develop an effective maintenance policy, it is therefore important to take into account such degradation effects in the assessment of the total operating cost. To answer this need, the present paper develops a continuous-time discrete-state Markov chain model to describe the deterioration stochastic process of a component, which is modeled as a multi-state system (MSS) where each discrete state is characterized by a degradation level (Lisnianski and Levitin, 2003). Unlike binary-state systems, the MSS has more than two levels of performance varying from perfect functioning to complete failure. An important feature of the proposed multi-state model lies in the fact that it takes into account side effect of degradation process into condition-based preventive maintenance optimization. The proposed model is extended to deal with multi-component multi-state series system. It is shown that preventive maintenance and side effect costs should not be optimized for each component individually, but from the perspective of the series system as a whole. 
The remainder of this paper is organised as follows. Section 2 presents the multi-state model proposed to describe the degradation stochastic process and the condition-based maintenance policy. Section 3 presents the method used to integrate side effect cost in preventively maintained degrading systems. Section 4 extends the model to deal with multi-component series system. Numerical examples are presented in Sections 3 and 4 to illustrate the proposed approach. Finally, Section 5 concludes the paper and provides some research perspectives.

\section{Multi-state Model and Preventive Maintenance Policy}

\subsection{Degradation Process}

During the system functioning, the operating cost increases along with the degraded level. This cost is a stochastic process, which depends on the degradation process. The system state space is discretized into several states characterized by different degradation levels. Even if systems can degrade continuously, to simplify the model, we assume that the degradation occurs in discrete steps. This is a good approximation if the system is discretized with enough steps to reflect the degradation. As time progresses, the system can go to the first degraded state, then to the second degraded state, and so forth, until reaching the complete failure state. The degradation process reflects the principle of the second law of thermodynamics, which states that there is a natural tendency of any isolated system to degenerate into a more disordered state, i.e., the entropy of an isolated system (disorder) increases with time.

Example Figure 1 sketches a degraded system representation with four states, which are illustrated by colors according to their degradation levels. There are gradual changes with time in state attributes, ranging from perfect performance (green) to the worst case (red). The failure state in red is reached when the degradation exceeds the specified threshold. The other states (yellow and orange) correspond to intermediate degradation levels. Figure 1 also illustrates the state transition graph representing such gradual and irreversible degradation process with the arrow of time.

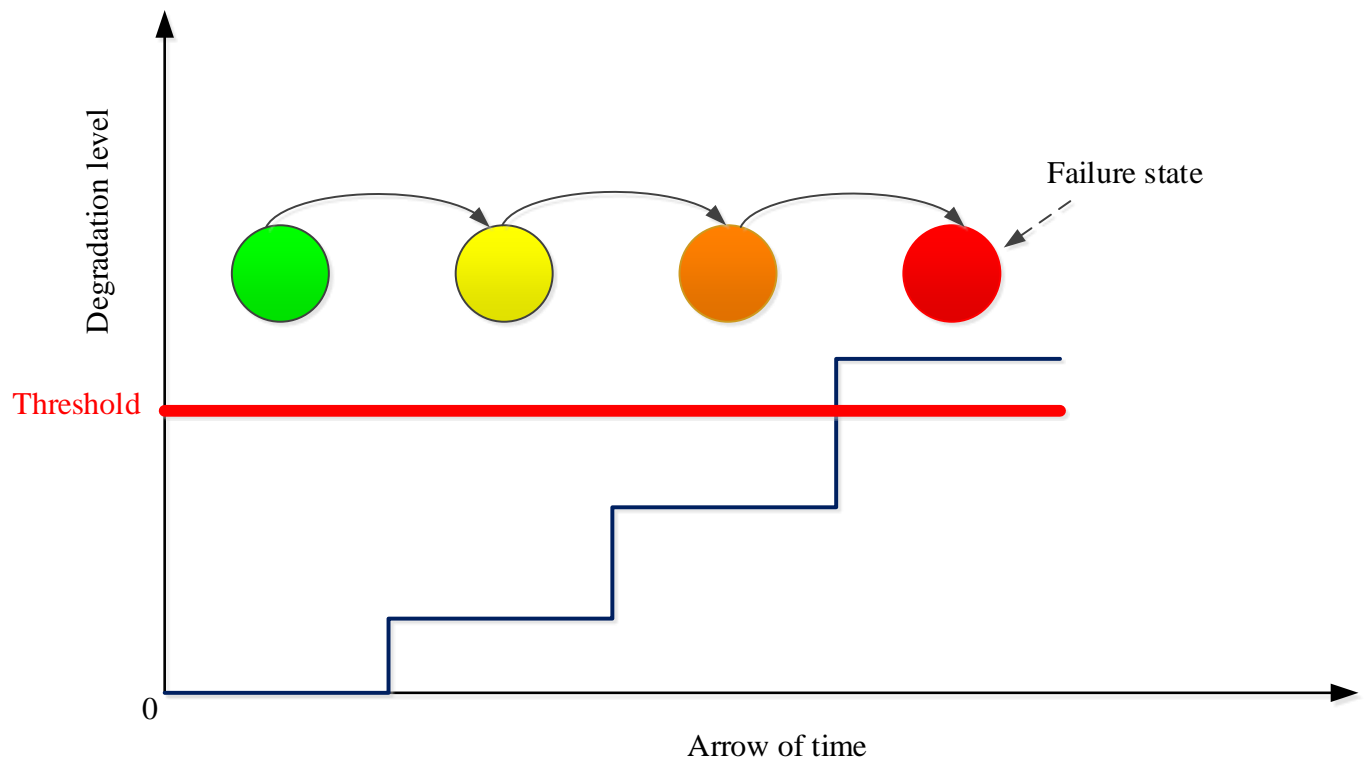

Figure 1. Illustration of a degraded system representation with four states. 


\subsection{Maintenance Policy Optimization}

We consider a condition-based preventive maintenance policy in which the degraded level of the system is continuously monitored. We assume that the condition of the system is always known exactly and that changes in its condition are observed immediately. The system states are divided into acceptable states and unacceptable states. The system entrance into any unacceptable state constitutes a failure. When the system reaches its last acceptable state, which is the failure threshold, a preventive maintenance (PM) is performed to restore the system to one of the previous higher performance states. In fact, if the degradation is not limited, the system's behavior will become unacceptable. To prevent this, the operator performs maintenance to avoid further degradation and to improve the condition of the system. Several kinds of PM actions are possible, varying from minor maintenance to major maintenance. A minor maintenance restores the system to the previous degraded state, while a major maintenance restores it to the "as good as new" state (i.e., the initial perfect functioning state). The cost and duration of the PM depends on the condition of the system when maintenance starts and its condition when it is completed. The start and end conditions are under the control of the maintenance operator. A PM strategy is defined as a pair composed of the last acceptable state before maintenance (the threshold failure state) and the operational state reached after maintenance. In the example of Figure 1, the last acceptable state is in orange, and two PM actions are possible to bring the system back, either to the perfect functioning state (in green), or to the intermediate state in yellow.

Furthermore, the system might fail randomly from any operational state, i.e., from the perfect functioning state as well as from any degraded acceptable state. This random and sudden event, called Poisson failure, occurs abruptly unlike the gradually worsening deterioration failures. If the system fails after a Poisson failure, it is minimally repaired. When the minimal repair (MR) is completed, the system returns to the condition it was in when the failure occurred and operations resume. There is a cost to minimal repair, and that cost depends on the condition of the machine at the time of failure.

Finally, we take into account catastrophic failures. This kind of failures terminate the system mission, and require replacement with a brand new system. We add transitions from each operational state to a catastrophic state from which we must perform a replacement by such new system.

The studied optimization problems consist in minimizing the total cost or maximizing the production rate. The decision variables are related to the selection of the best PM strategy. The total cost is the sum of the maintenance costs and the operating costs. The maintenance costs are the MR cost, the PM cost, and the replacement cost. The operating costs are due to degradation and capture the side effect costs. Using our methodology, the selected PM strategy takes into account the tradeoff between maintenance and operating costs.

\subsection{Continuous-time Markov Chain Model}

To develop the multi-state model representing the degraded system subjected to the maintenance policy described above, we assume that all probability distributions are exponential and all the rates are known. The resulting continuous-time Markov chain model is represented by Figure 2. The meanings of states and transitions and all the notation used in this figure are presented in Table 1. 


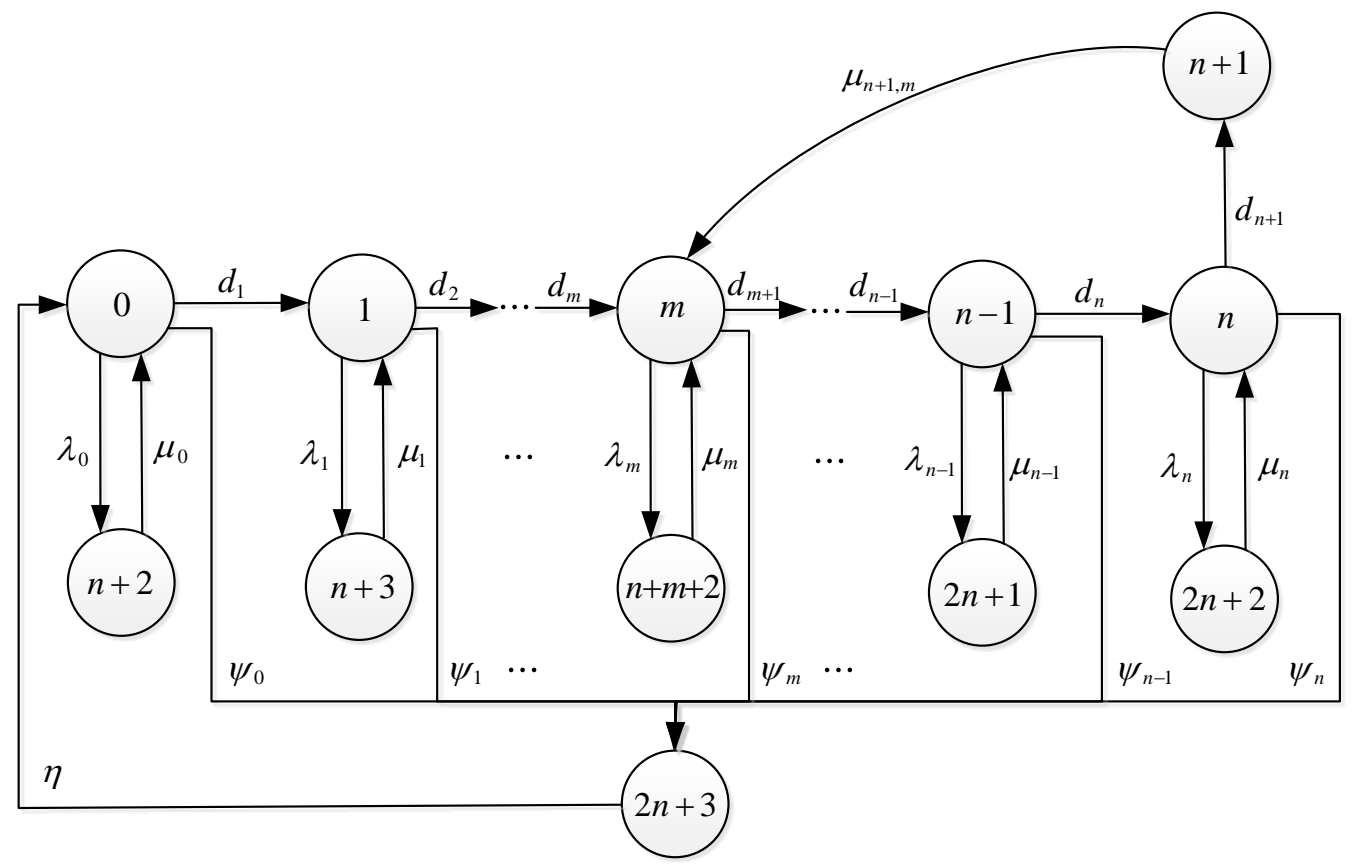

Figure 2. State-transition diagram for a multi-state degraded system subjected to condition-based preventive maintenance.

Table 1. Notation used in figure 2.

\begin{tabular}{|l|l|}
\hline \multicolumn{2}{|c|}{ States } \\
\hline Operational: 0 to $n$ & Non-operational: $n+1$ to $2 n+3$ \\
\hline Perfect (as good as new): State 0 & Preventive maintenance state: $n+1$ \\
\hline Degraded: States 1 to $n$; State $m$ is reached after PM & Minimal repair states: $n+2$ to $2 n+2$ \\
\hline Last acceptable state: $n$ & Catastrophic state: $2 n+3$ \\
\hline & \multicolumn{1}{c|}{ Transitions } \\
\hline Degradation rates & $d_{1}, \ldots, d_{n+1}$ \\
\hline Preventive maintenance rates & $\mu_{n+1, m}$ \\
\hline Failure rates & $\lambda_{0} \ldots, \lambda_{n}$ \\
\hline Minimal repair rates & $\mu_{0} \ldots, \mu_{n}$ \\
\hline Catastrophic failure rates & $\psi_{0} \ldots, \psi_{n}$ \\
\hline Renewal rate (after catastrophic failure) & $\eta$ \\
\hline
\end{tabular}

\section{Integrating Side Effect Costs}

\subsection{Operating Cost}

Incurred when the system is operational, the operating cost is related to side effects. The following notation is used:

$i \quad$ Operational state index, $i=0,1,2, \ldots, n$

$\rho_{i} \quad$ Production rate in state $i$

$\phi_{i} \quad$ Fraction of non-conforming items produced when the system is in state $i$ 
$q_{i} \quad$ Cost incurred by producing one non-conforming item in state $i$

$C_{Q} \quad$ Cost of producing non-conforming items

$S_{i} \quad$ Other side effect cost incurred in state $i$

$P_{i} \quad$ Steady-state probability of the system being in state $i$

For each operational state $i$, the fraction of conforming items is $\left(1-\phi_{i}\right)$ and the production rate of conforming items is given by $\rho_{i}\left(1-\phi_{i}\right)$. The cost of producing non-conforming items in state $i$ is the product of the production rate of non-conforming items $\rho_{i} \phi_{i}$ and the unitary cost $q_{i}$. The expected total cost of producing non-conforming items is

$C_{Q}=\sum_{i=1}^{n} q_{i} \rho_{i} \phi_{i} P_{i}$

The expected total side effect cost is

$S_{E}=\sum_{i=1}^{n} S_{i} P_{i}$

As the system degradation increases with time, we have in general $\rho_{1} \geq \rho_{2} \geq \ldots \geq \rho_{n} \geq 0$; $0 \leq \phi_{1} \leq \phi_{2} \leq \ldots \leq \phi_{n} ; 0 \leq q_{1} \leq q_{2} \leq \ldots \leq q_{n} ;$ and $0 \leq S_{1} \leq S_{2} \leq \cdots \leq S_{n}$.

The total operating cost, $C_{O}$, is then given by

$C_{O}=\sum_{i=1}^{n}\left(q_{i} \rho_{i} \phi_{i}+S_{i}\right) P_{i}$

\subsection{Maintenance Cost}

To estimate the expected maintenance cost, the following additional notation is used:

$c_{(n+1, m)}$ Cost of one preventive maintenance performed from state $n+1$ to state $m$

$r_{i} \quad$ Cost of a minimal repair due to a Poisson failure in state $i$

$c_{\eta} \quad$ Cost of a repair due to a catastrophic failure

The maintenance cost is the sum of the PM cost, the MR cost, and the cost of repair after catastrophic failures. Thus, the steady-state expected total maintenance cost, $C_{M}(n+1, m)$, depends on PM strategy defined by the threshold state $n+1$ and the state $m$ reached after PM. We have

$C_{M}(n+1, m)=c_{(n+1, m)} P_{n+1}+c_{\eta} P_{2 n+3}+\sum_{i=0}^{n} r_{i} P_{i+2+n}$

\subsection{Cost Minimization}

The objective function to be minimized is the total cost, $C_{T}(n+1, m)$, which is the sum of the maintenance and the operating costs. Using the above equations, we have 
$C_{T}(n+1, m)=c_{(n+1, m)} P_{n+1}+c_{\eta} P_{2 n+3}+\sum_{i=0}^{n} r_{i} P_{i+2+n}+\sum_{i=1}^{n}\left(q_{i} \rho_{i} \phi_{i}+S_{i}\right) P_{i}$

The goal of the optimization is to select the minimal cost PM strategy. This allows for the best trade-off between maintenance costs, quality costs and other side effect costs. Given the component parameters, the solution approach consists in comparing all the PM strategies by evaluating the total cost expressed in Equation (5). The decision variables are the threshold state $n+1$ and the state $m$ reached after PM. For a given PM strategy $(n+1, m)$, it is required to evaluate the probabilities in the Markov chain model of Figure 2. For this, the steady-state Chapman-Kolmogorov equations are represented as

$$
\left[\begin{array}{cccccccccccccccc}
-\lambda_{0}-\psi_{0}-d_{1} & d_{1} & \cdots & 0 & \cdots & 0 & 0 & 0 & \lambda_{0} & 0 & \cdots & 0 & \cdots & 0 & 0 & \psi_{0} \\
0 & -\lambda_{1}-\psi_{1}-d_{2} & d_{2} & 0 & \cdots & 0 & 0 & 0 & 0 & \lambda_{1} & \cdots & 0 & \cdots & 0 & 0 & \psi_{1} \\
\vdots & \ddots & \ddots & \ddots & \vdots & \vdots & \vdots & \vdots & \vdots & \vdots & \ddots & \vdots & \ddots & \vdots & \vdots & \vdots \\
0 & 0 & \ddots & -\lambda_{m}-\psi_{m}-d_{m+1} & d_{m+1} & 0 & 0 & 0 & 0 & 0 & \cdots & \lambda_{m} & \cdots & 0 & 0 & \psi_{m} \\
\vdots & \vdots & \ddots & \vdots & \ddots & \ddots & \vdots & \vdots & \vdots & \vdots & \ddots & \vdots & \ddots & \vdots & \vdots & \vdots \\
0 & 0 & \cdots & 0 & \cdots & -\lambda_{n-1}-\psi_{n-1}-d_{n} & d_{n} & 0 & 0 & 0 & \cdots & 0 & \cdots & \lambda_{n-1} & 0 & \psi_{n-1} \\
0 & 0 & \cdots & 0 & \cdots & 0 & -\lambda_{n}-\psi_{n}-d_{n+1} & d_{n+1} & 0 & 0 & \cdots & 0 & \cdots & 0 & \lambda_{n} & \psi_{n} \\
0 & 0 & \cdots & \mu_{n+1, m} & \cdots & 0 & 0 & -\mu_{n+1, m} & 0 & 0 & \cdots & 0 & \cdots & 0 & 0 & 0 \\
\mu_{0} & 0 & \cdots & 0 & \cdots & 0 & 0 & 0 & -\mu_{0} & 0 & \cdots & 0 & \cdots & 0 & 0 & 0 \\
0 & \mu_{1} & \cdots & 0 & \cdots & 0 & 0 & 0 & 0 & -\mu_{1} & \cdots & 0 & \cdots & 0 & 0 & 0 \\
\vdots & \vdots & \ddots & \vdots & \vdots & \vdots & \vdots & \vdots & \vdots & \vdots & \ddots & \vdots & \vdots & \vdots & \vdots & \vdots \\
0 & 0 & \cdots & \mu_{m} & \cdots & 0 & 0 & 0 & 0 & 0 & \cdots & -\mu_{m} & \cdots & 0 & 0 & 0 \\
\vdots & \vdots & \vdots & \vdots & \ddots & \vdots & \vdots & \vdots & \vdots & \vdots & \vdots & \vdots & \ddots & \vdots & \vdots & \vdots \\
0 \\
0
\end{array}\right.
$$

and the normalization equation is

$\sum_{i=0}^{2 n+3} P_{i}=1$

The last row in Equation (6) is substituted by the normalization Equation (7) in order to obtain a system of $(2 n+4)$ linear equations with $(2 n+4)$ unknowns, which is solved to calculate the steadystate probabilities $P_{i}$.

\subsection{Numerical Example}

\subsubsection{Input Data}

Let consider a multi-state degraded system for which the state-transition diagram is sketched in Figure 3. This system is subjected to a condition-based PM as in the proposed model. The statetransition diagram can be sketched for all possible threshold states $(1,2,3,4)$. For example, Figure 4 presents this diagram when the threshold state is $n=3$. Dotted lines indicate transitions to be selected: one PM action will be selected corresponding to one of the three possible transition rates $\left(\mu_{3,2}, \mu_{3,1}\right.$, or $\left.\mu_{3,0}\right)$. 


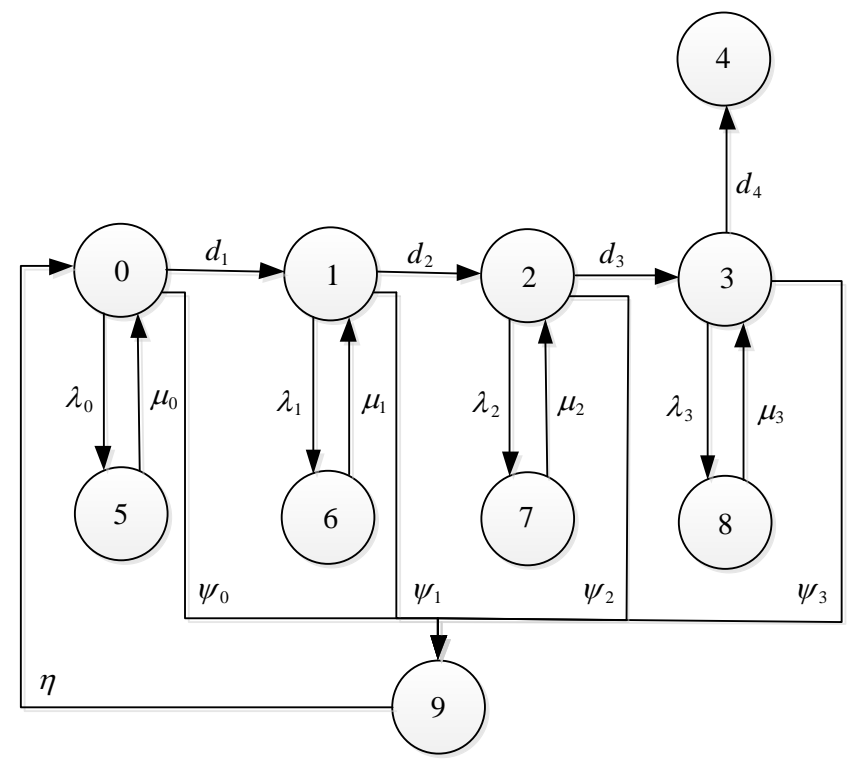

Figure 3. Example of multi-state degraded system.

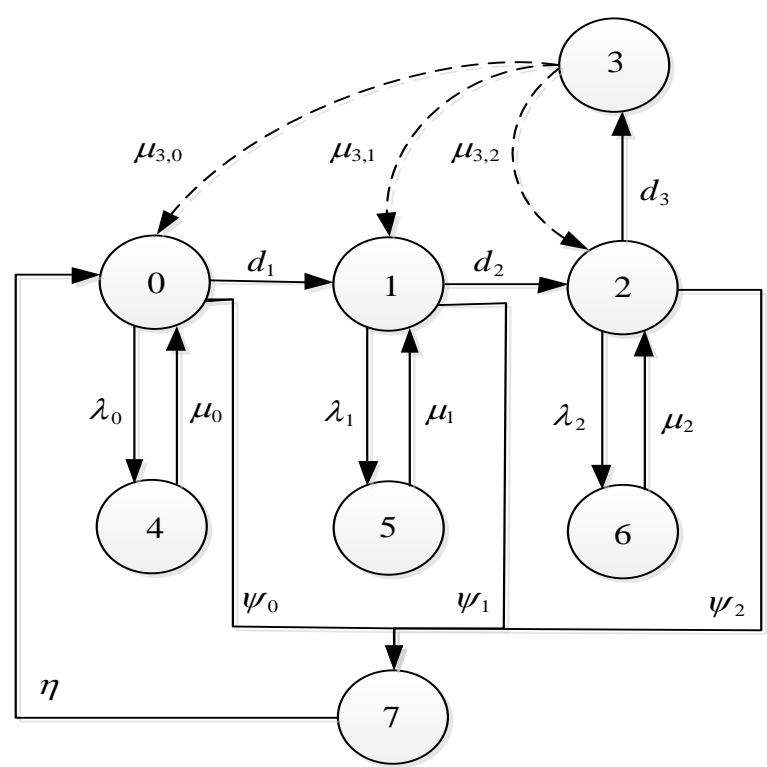

Figure 4. Example of multi-state system subjected to PM.

The input data required for the optimization problem studied in this example are provided in Tables 2-4. Table 2 presents different transition rates and repair costs, while Table 3 specifically presents the PM transition rates with the corresponding costs. Table 4 presents the state attributes, namely production rates, percentages and costs of non-conforming products, and costs related to energy 
consumption and emission. All costs are expressed in $\$$ per day, transition rates are per day, and production rates are in parts per day.

Table 2. Transition rates.

\begin{tabular}{|l|l|}
\hline Degradation rates $\left(d_{1}, d_{2}, d_{3}, d_{4}\right)$ & $(0.016,0.017,0.018,0.019)$ \\
\hline Failure rates $\left(\lambda_{0}, \lambda_{1}, \lambda_{2}, \lambda_{3}\right)$ & $(0.031,0.033,0.036,0.039)$ \\
\hline Minimal repair rates and costs $\left(\mu_{i}, r_{i}\right)$ & $(0.2,4000),(0.2,5000),(0.2,5500),(0.2,6000)$ \\
\hline Catastrophic failure rates $\left(\psi_{0}, \psi_{1}, \psi_{2}, \psi_{3}\right)$ & $\left(10^{-9}, 10^{-9}, 10^{-9}, 10^{-9}\right)$ \\
\hline Renewal rate and cost after catastrophic failure: $\left(\eta, c_{\eta}\right)$ & $(0.033,130000)$ \\
\hline
\end{tabular}

Table 3. PM transition rates with the corresponding costs: $\left(\mu_{(n+1, m)}, c_{(n+1, m)}\right)$.

\begin{tabular}{|c|c|c|c|c|c|}
\hline & \multicolumn{5}{|c|}{ Threshold state $(\boldsymbol{n + 1})$} \\
\hline \multirow{4}{*}{ State reached after PM $(m)$} & & 1 & 2 & 3 & 4 \\
\cline { 2 - 6 } & 0 & $(0.1,14000)$ & $(0.09,14500)$ & $(0.085,14600)$ & $(0.08,14700)$ \\
\cline { 2 - 6 } & 1 & - & $(0.1,13000)$ & $(0.09,15000)$ & $(0.05,15100)$ \\
\cline { 2 - 6 } & 2 & - & - & $(0.1,10000)$ & $(0.09,14000)$ \\
\cline { 2 - 6 } & 3 & - & - & - & $(0.1,8000)$ \\
\hline
\end{tabular}

Table 4. State attributes.

\begin{tabular}{|l|l|}
\hline Production rates $\left(\rho_{0}, \rho_{1}, \rho_{2}, \rho_{3}\right)$ & $(1500,1450,1400,1350)$ \\
\hline Fractions of non-conforming products $\left(\phi_{0}, \phi_{1}, \phi_{2}, \phi_{3}\right)$ & $(0,0.05,0.06,0.07)$ \\
\hline Cost of producing one non-conforming item $\left(q_{0}, q_{1}, q_{2}, q_{3}\right)$ & $(-, 2,3,4)$ \\
\hline Side effect cost $\left(S_{0}, S_{1}, S_{2}, S_{3}\right)$ & $(-, 213,215,330)$ \\
\hline
\end{tabular}

\subsubsection{Results and Discussion}

The results obtained by our optimization model are presented in Table 5. Figure 5 sketches a graph representing the total cost for each PM strategy. Rounding numbers to their nearest whole numbers, the best PM strategy has a total cost of 1656 \$ per day, a PM cost of 788 \$ per day, and an operating cost of $216 \$$ per day. This strategy recommends that when the system reaches the degradation threshold level characterized by State 3, it should be preventively maintained to occupy the perfect initial state.

If the optimization model only considers maintenance costs (without taking into account the operating costs related to quality, energy and emissions), the results in Table 5 suggest strategy (4, 0 ) instead of strategy $(3,0)$. This results in a loss of about $19 \$$ per day. This is due to the fact that the system degradation is allowed, which causes undesirable operating costs related to the production of non-conforming items and additional energy and emission costs. These results show that the proposed model integrating side effect costs provide a better solution than a model ignoring such side effects costs. 
Table 5. Optimization model results.

\begin{tabular}{|c|c|c|c|c|c|c|c|c|}
\hline $\begin{array}{c}\text { PM } \\
\text { strategy } \\
(\boldsymbol{n + 1 , m})\end{array}$ & $\begin{array}{c}\text { Quality } \\
\text { cost }\end{array}$ & $\begin{array}{c}\text { Side effect } \\
\text { cost }\end{array}$ & $\begin{array}{c}\text { Operating } \\
\text { cost }\end{array}$ & $\begin{array}{c}\text { Repair } \\
\text { cost }\end{array}$ & $\begin{array}{c}\text { PM } \\
\text { cost }\end{array}$ & $\begin{array}{c}\text { Maintenance } \\
\text { cost }\end{array}$ & $\begin{array}{c}\text { Total } \\
\text { cost }\end{array}$ & $\begin{array}{c}\text { Production } \\
\text { rate } \\
\text { (conforming) }\end{array}$ \\
\hline$(1,0)$ & 0 & 0 & 0 & 471.483 & 1703.422 & 2174.905 & 2174.905 & 1140.684 \\
\hline$(2,0)$ & 56.178 & 82.524 & 138.702 & 574.857 & 1061.142 & 1635.999 & 1774.701 & 1151.167 \\
\hline $\mathbf{( 3 , 0 )}$ & 103.328 & 112.246 & 215.575 & 652.547 & 787.777 & 1440.324 & $\mathbf{1 6 5 5 . 8 9 8}$ & 1136.919 \\
\hline$(\mathbf{4 , 0})$ & 150.618 & 148.552 & 299.17 & 723.756 & 652.366 & $\mathbf{1 3 7 6 . 1 2 2}$ & $\mathbf{1 6 7 5 . 2 9 2}$ & 1114.682 \\
\hline$(2,1)$ & 108.614 & 159.551 & 268.165 & 617.978 & 1655.431 & 2273.408 & 2541.573 & 1031.835 \\
\hline$(3,1)$ & 155.165 & 168.557 & 323.723 & 713.032 & 1147.873 & 1860.905 & 2184.628 & 1061.603 \\
\hline$(4,1)$ & 195.521 & 192.84 & 388.361 & 760.939 & 1391.835 & 2152.774 & 2541.135 & 1014.931 \\
\hline$(3,2)$ & 185.294 & 158.088 & 343.382 & 727.941 & 1323.529 & 2051.471 & 2394.853 & 967.647 \\
\hline$(4,2)$ & 242.866 & 210.036 & 452.902 & 835.324 & 1114.603 & 1949.927 & 2402.828 & 997.339 \\
\hline$(4,3)$ & 272.924 & 238.267 & 511.191 & 844.765 & 1097.473 & 1942.238 & 2453.43 & 906.498 \\
\hline
\end{tabular}

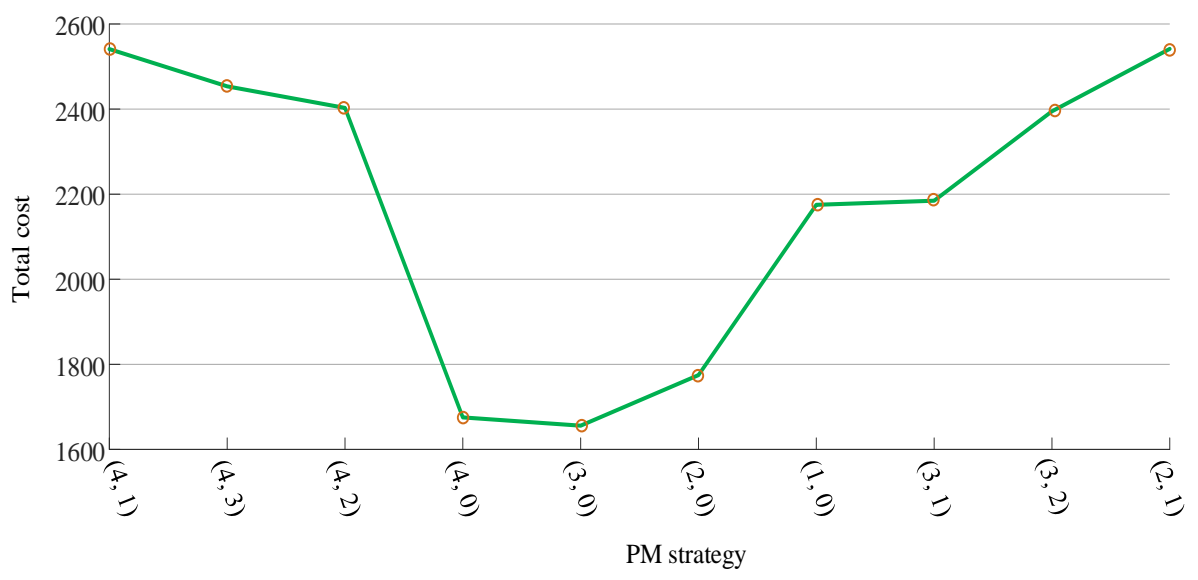

Figure 5. Total cost for each PM strategy.

\section{Maintenance Optimization of Series Multi-state Degraded Systems}

In this section, we consider a multi-component series system. Each component is multi-state and is subjected to condition-based preventive maintenance. An optimization model is formulated to select the best preventive maintenance strategy for each component. Two optimization models are solved. In the first model, the objective is to define the optimal maintenance policy for each component, so that the total cost is minimized. The system performance is represented by the production rate of conforming items. In the second model, the objective function corresponds to the system production rate maximization.

\subsection{Optimization Model}

Let consider a system consisting of $K$ components connected in series. We indicate by $k(k=1,2$, $\ldots, g$ ) the index for components. As illustrated by Figure 6, each component is represented according to the multi-state model presented in the previous sections.

Assuming that the components are independent, the bottleneck of the series system should be identified firstly due to the characteristics of unbalanced systems. For the bottleneck component, it 
may operate at the full machine speed, $P r_{b n}$. In contrast, other components operate at reduced machine speed. In this context, the system total cost, $T C$, is the sum of all components maintenance and operating costs denoted by $T C_{O}$ and $T C_{M}$ (respectively):

$$
\begin{aligned}
\operatorname{Pr}_{b n} & =\min \left\{\operatorname{Pr}_{1}, P r_{2}, \ldots, P r_{g}\right\} \\
& =\min \left\{\sum_{i=1}^{n} \rho_{i}^{1} P_{i}^{1}, \sum_{i=1}^{n} \rho_{i}^{2} P_{i}^{2}, \ldots, \sum_{i=1}^{n} \rho_{i}^{g} P_{i}^{g}\right\} \\
T C & =T C_{O}+T C_{M} \\
& =\sum_{i=1}^{n}\left(q_{i}^{b n} \rho_{i}^{b n} \phi_{i}^{b n}+S_{i}^{b n}\right) P_{i}^{b n}+\sum_{k=1}^{g-1} \sum_{i=1}^{n}\left(\frac{P r_{b n}}{P r_{k}} \rho_{i}^{k} \phi_{i}^{k} q_{i}^{k}+S_{i}^{k}\right) P_{i}^{k}+\sum_{k=1}^{g} \sum_{i=0}^{n} r_{i}^{k} P_{i+2+n}^{k}+\sum_{k=1}^{g}\left(c_{(n+1, n)}^{k} P_{n+1}^{k}+c_{\eta}^{k} P_{2 n+3}^{k}\right)
\end{aligned}
$$

The performance constraint may require that the conforming items production rate of the system, $\pi$, should be equal or higher to a pre-specified minimum level $\pi \geq \pi_{\min }$ :

$\pi \geq \pi_{\min }$

Multi-component series system

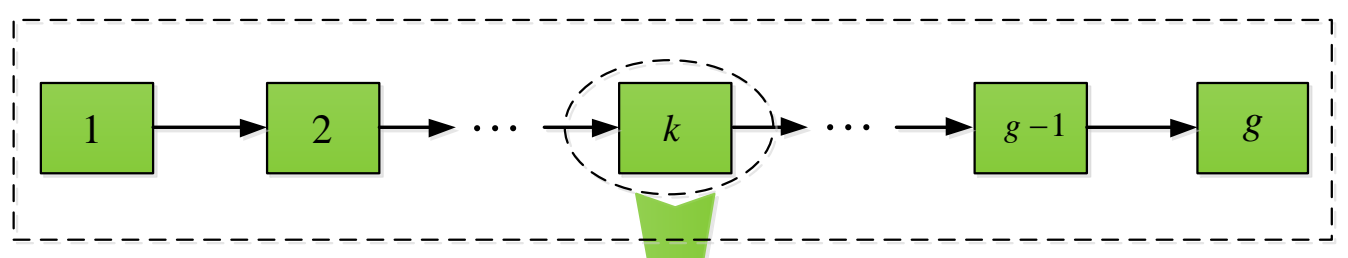

Component

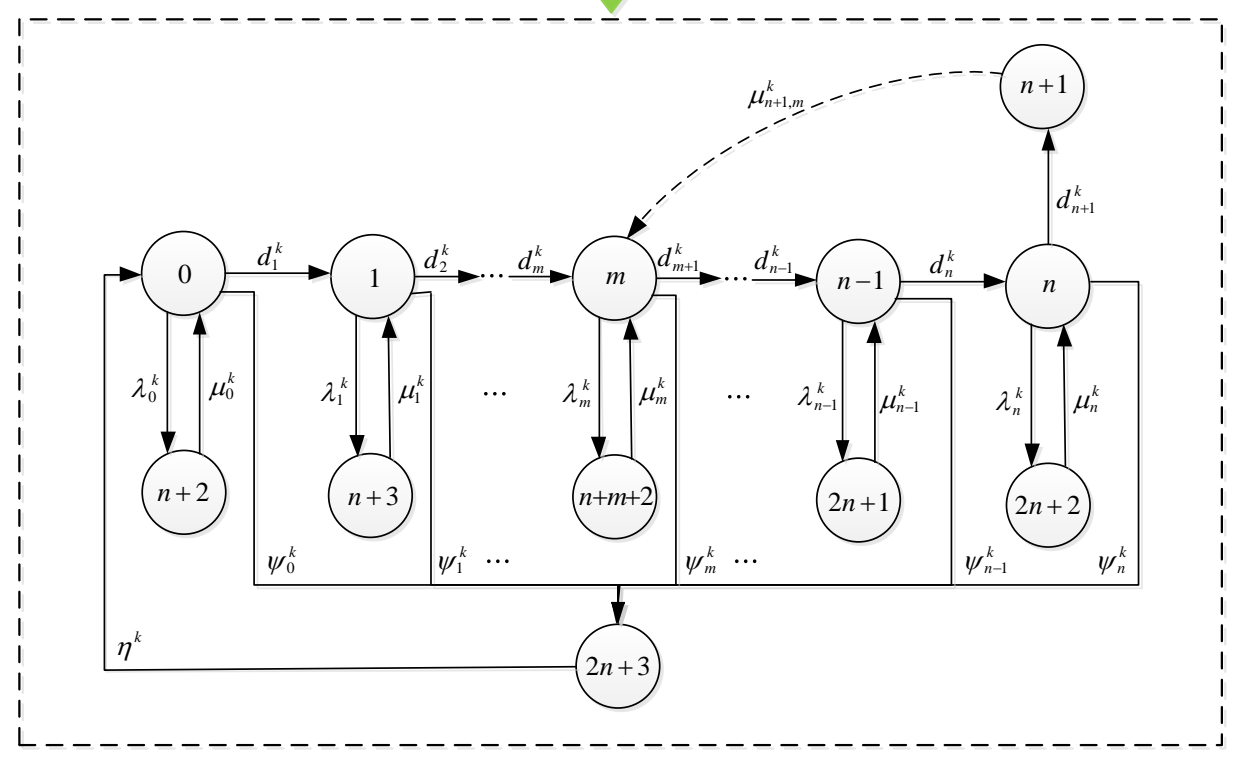

Figure 6. Multi-state series system. 
As the components are connected in series, the component with the least performance becomes the bottleneck of the system. This component, therefore, defines the total system production rate. The decision variables are related to the selection of the best PM strategy for all components by selecting for all components the threshold states and the states reached after PM. Because of the system constraint (10), it is important to optimize preventive maintenance from the perspective of the series system as a whole. Using our model, it is also possible to consider the maximization of the system production rate as the objective function under eventual budget constraints.

\subsection{Numerical Results}

Consider a series system with two components. The input data for each component are given in Table 6. Furthermore, three PM strategies are possible for each component, namely strategies (4, $1),(4,2)$ and $(4,3)$ with transition rates and costs $(0.05,15100),(0.09,14000)$ and $(0.1,8000)$, respectively. All data are the same for Components 1 and 2, except for the production rates. From these production rates, we remark that Component 2 is less efficient than Component 1.

Table 6. Input data of the two components in the series system.

\begin{tabular}{|l|l|}
\hline Degradation rates & $\begin{array}{l}(0.016,0.017,0.018,0.019) \\
(0.016,0.017,0.018,0.019)\end{array}$ \\
\hline Failure rates & $\begin{array}{l}(0.031,0.033,0.036,0.039) \\
(0.031,0.033,0.036,0.039)\end{array}$ \\
\hline Minimal repair rates and costs & $\begin{array}{l}(0.2,4000),(0.2,5000),(0.2,5500),(0.2,6000) \\
(0.2,4000),(0.2,5000),(0.2,5500),(0.2,6000)\end{array}$ \\
\hline Catastrophic failures & - \\
\hline \multicolumn{2}{|c|}{ State attributes } \\
\hline Production rates & $\begin{array}{l}(1500,1450,1400,1350) \\
(1200,1180,1100,1000)\end{array}$ \\
\hline Non-conforming products & - \\
\hline \multirow{2}{*}{ Side effect costs } & $\begin{array}{l}(-, 213,215,330) \\
(-, 213,215,330)\end{array}$ \\
\hline
\end{tabular}

Table 7 summarizes the results obtained using our model when the objective is to maximize the production rate. Two cases are considered.

Case 1: Each Component is Individually Optimized

The best PM strategy is $(4,1)$ for both components. Rounding numbers to their nearest whole numbers, the system total cost is $4692 \$$ per day and the system production rate is 844 parts per day.

\section{Case 2: The Optimization Considers the Series System as a Whole}

In this case, the best PM strategy is $(4,1)$ for Component 2 to ensure a maximum system production rate of 844 parts per day. For Component 1, all PM strategies will ensure this maximum system production rate imposed by the bottleneck Component 2. Therefore, it is better to select strategy $(4,2)$ as it has the lowest cost. Rounding numbers to their nearest whole numbers, the system total cost is $4506 \$$ per day and the system production rate is 844 parts per day.

We remark that the maximum production rate is reached at a lower cost when the optimization considers the side effects costs in the series system as a whole (4506 \$ per day instead of 4692 \$ per day). We also observe that Component 2 is less efficient is subjected to the highest PM level 
$(4,1)$, while it is sufficient to undergo PM strategy $(4,2)$ for Component 1 , since Component 2 is the bottleneck in this series system.

Table 7. Results for components 1 and 2.

\begin{tabular}{|c|c|c|c|c|c|c|}
\hline \multicolumn{7}{|c|}{ Component 1 } \\
\hline PM strategy & Operating cost & Repair cost & PM cost & Maintenance cost & Total cost & Production rate \\
\hline $\mathbf{( 4 , 1 )}$ & 192.84 & 760.939 & 1391.835 & 2152.774 & 2345.614 & $\mathbf{1 0 7 9 . 0 1 6}$ \\
\hline$(4, \mathbf{2})$ & 210.036 & 835.324 & 1114.603 & 1949.927 & $\mathbf{2 1 5 9 . 9 6 2}$ & 1066.415 \\
\hline$(4,3)$ & 238.267 & 844.765 & 1097.473 & 1942.238 & 2180.505 & 974.729 \\
\hline \multicolumn{7}{|c|}{ Component 2 } \\
\hline PM strategy & Operating cost & Repair cost & PM cost & Maintenance cost & Total cost & Production rate \\
\hline$(4, \mathbf{1})$ & 192.84 & 760.939 & 1391.835 & 2152.774 & 2345.614 & $\mathbf{8 4 4 . 1 0 9}$ \\
\hline$(\mathbf{4 , 2})$ & 210.036 & 835.324 & 1114.603 & 1949.927 & $\mathbf{2 1 5 9 . 9 6 2}$ & 815.001 \\
\hline$(4,3)$ & 238.267 & 844.765 & 1097.473 & 1942.238 & 2180.505 & 722.022 \\
\hline
\end{tabular}

\section{Conclusions}

This paper develops a continuous-time discrete-state Markov chain model to characterize the deterioration stochastic process. It considers a multi-state system with each discrete state characterized by a degradation level. The proposed multi-state model takes into account side effect costs. Optimization models including different constraints and objective functions are discussed. The results have confirmed the importance of considering side effects costs when optimizing the choice of maintenance policy. The proposed multi-state characterization has established a building model for a single component. After this, the model has been extended to deal with series systems, and it was shown that preventive maintenance and side effect costs should not be optimized for each component individually, but from the perspective of the series system as a whole.

Future work will consider condition-based preventive maintenance optimization of series-parallel multi-component systems. For large systems, heuristic methods would be required to solve the resulting combinatorial optimization problem.

\section{Conflict of Interest}

The authors confirm that there is no conflict of interest to declare for this publication.

\section{Acknowledgments}

The authors would like to thank the China Scholarship Council (CSC) for supporting funding of the first author.

\section{References}

Abdel-Hameed, M. (1975). A gamma wear process. IEEE Transactions on Reliability, 24(2), 152-153.

An, Y., Chen, X., Zhang, J., \& Li, Y. (2020). A hybrid multi-objective evolutionary algorithm to integrate optimization of the production scheduling and imperfect cutting tool maintenance considering total energy consumption. Journal of Cleaner Production, 268, 121540.

Ba, K., Dellagi, S., Rezg, N., \& Erray, W. (2016). Joint optimization of preventive maintenance and spare parts inventory for an optimal production plan with consideration of $\mathrm{CO} 2$ emission. Reliability Engineering \& System Safety, 149, 172-186. 
Barlow, R., \& Hunter, L. (1960). Optimum preventive maintenance policies. Operations Research, 8(1), 90100.

Eda, K., Iga, A., \& Matsuoka, M. (1980). Degradation mechanism of non-ohmic zinc oxide ceramics. Journal of Applied Physics, 51(5), 2678-2684.

Fakher, H.B., Nourelfath, M., \& Gendreau, M. (2017). A cost minimisation model for joint production and maintenance planning under quality constraints. International Journal of Production Research, 55(8), 2163-2176.

Fitouhi, M.C., Nourelfath, M., \& Gershwin, S.B. (2017). Performance evaluation of a two-machine line with a finite buffer and condition-based maintenance. Reliability Engineering \& System Safety, 166, 61-72.

Flory, J.A., Kharoufeh, J.P., \& Abdul-Malak, D.T. (2015). Optimal replacement of continuously degrading systems in partially observed environments. Naval Research Logistics, 62(5), 395-415.

Gouiaa-Mtibaa, A., Dellagi, S., Achour, Z., \& Erray, W. (2018). Integrated maintenance-quality policy with rework process under improved imperfect preventive maintenance. Reliability Engineering \& System Safety, 173, 1-11.

Hajej, Z., Rezg, N., \& Gharbi, A. (2017). Joint optimization of production and maintenance planning with an environmental impact study. The International Journal of Advanced Manufacturing Technology, 93(14), 1269-1282.

Hoang, A., Do, P., \& Iung, B. (2017). Energy efficiency performance-based prognostics for aided maintenance decision-making: Application to a manufacturing platform. Journal of Cleaner Production, $142,2838-2857$.

Jeon, H.W., Taisch, M., \& Prabhu, V.V. (2015). Modelling and analysis of energy footprint of manufacturing systems. International Journal of Production Research, 53(23), 7049-7059.

Kallen, M.J., \& Noortwijk, J.M.V. (2005). Optimal maintenance decisions under imperfect inspection. Reliability Engineering \& System Safety, 90(2-3), 177-185.

Kang, R., Gong, W., \& Chen, Y. (2020). Model-driven degradation modeling approaches: Investigation and review. Chinese Journal of Aeronautics, 33(4), 1137-1153.

Kim, M.S., \& Sarkar, B. (2017). Multi-stage cleaner production process with quality improvement and lead time dependent ordering cost. Journal of Cleaner Production, 144, 572-590.

Lam, J.Y.J., \& Banjevic, D. (2015). A myopic policy for optimal inspection scheduling for condition based maintenance. Reliability Engineering \& System Safety, 144, 1-11.

Liao, G.L. (2013). Optimal economic production quantity policy for randomly failing process with minimal repair, backorder and preventive maintenance. International Journal of Systems Science, 44(9), 16021612.

Lisnianski, A., \& Levitin, G. (2003). Multi-state system reliability: assessment, optimization and applications. World scientific, Singapore.

Liu, B., Wu, S., Xie, M., \& Kuo, W. (2017). A condition-based maintenance policy for degrading systems with age-and state-dependent operating cost. European Journal of Operational Research, 263(3), 879887.

Liu, B., Xie, M., \& Kuo, W. (2016). Reliability modeling and preventive maintenance of load-sharing systemswith degrading components. Iie Transactions, 48(8), 699-709.

Liu, B., Xu, Z., Xie, M., \& Kuo, W. (2014). A value-based preventive maintenance policy for multicomponent system with continuously degrading components. Reliability Engineering \& System Safety, 132, 83-89. 
Marais, K.B., \& Saleh, J.H. (2009). Beyond its cost, the value of maintenance: an analytical framework for capturing its net present value. Reliability Engineering \& System Safety, 94(2), 644-657.

Nourelfath, M., Nahas, N., \& Ben-Daya, M. (2016). Integrated preventive maintenance and production decisions for imperfect processes. Reliability Engineering \& System Safety, 148, 21-31.

Pan, E.S., \& Chen, Z. (2015). Review of degradation model for high reliability products. Industrial Engineering and Management, 13, 1-6.

Peng, C.Y. (2015). Inverse Gaussian processes with random effects and explanatory variables for degradation data. Technometrics, 57(1), 100-111.

Rosqvist, T., Laakso, K., \& Reunanen, M. (2009). Value-driven maintenance planning for a production plant. Reliability Engineering \& System Safety, 94(1), 97-110.

Singh, G., Kumar, T.Ch.A., \& Naikan, V.N.A. (2019). Efficiency monitoring as a strategy for cost effective maintenance of induction motors for minimizing carbon emission and energy consumption. Reliability Engineering \& System Safety, 184, 193-201.

Tagaras, G. (1988). An integrated cost model for the joint optimization of process control and maintenance. Journal of the Operational Research Society, 39(8), 757-766.

Wang, X., \& Xu, D. (2010). An inverse Gaussian process model for degradation data. Technometrics, 52(2), $188-197$.

Wu, X., \& Ryan, S.M. (2010). Value of condition monitoring for optimal replacement in the proportional hazards model with continuous degradation. Iie Transactions, 42(8), 553-563.

Xiang, Y. (2013). Joint optimization of $\bar{X}$ control chart and preventive maintenance policies: a discrete-time Markov chain approach. European Journal of Operational Research, 229(2), 382-390.

Ye, Z.S., Chen, N., \& Shen, Y. (2015). A new class of Wiener process models for degradation analysis. Reliability Engineering \& System Safety, 139, 58-67.

Ye, Z.S., Wang, Y., Tsui, K.L., \& Pecht, M. (2013). Degradation data analysis using Wiener processes with measurement errors. IEEE Transactions on Reliability, 62(4), 772-780.

Ye, Z.S., \& Xie, M. (2015). Stochastic modelling and analysis of degradation for highly reliable products. Applied Stochastic Models in Business and Industry, 31(1), 16-32.

Yeung, T.G., Cassady, C.R., \& Schneider, K. (2007). Simultaneous optimization of [Xbar] control chart and age-based preventive maintenance policies under an economic objective. Iie Transactions, 40(2), 147159.

Yin, M.L., Angus, J.E., \& Trivedi, K.S. (2013). Optimal preventive maintenance rate for best availability with hypo-exponential failure distribution. IEEE Transactions on Reliability, 62(2), 351-361.

Zhou, B., Qi, Y., \& Liu, Y. (2020). Proactive preventive maintenance policy for buffered serial production systems based on energy saving opportunistic windows. Journal of Cleaner Production, 253, 119791. 